\title{
Incidence of Bloodstream Infections in Patients with COVID-19: A Retrospective Cohort Study of Risk Factors and Outcomes
}

Claudia Villatoro Santos ( $\sim$ Claudia.Villatoro.santos@ascension.org )

St. John Hospital \& Medical Center

Elisa Akagi Fukushima

St. John Hospital \& Medical Center

Wei Zhao

St. John Hospital \& Medical Center

\section{Mamta Sharma}

St. John Hospital \& Medical Center

\section{Dima Youssef}

St. John Hospital \& Medical Center

\section{Susan Spzunar}

St. John Hospital \& Medical Center

\section{Miriam Levine}

St. John Hospital \& Medical Center

\section{Louis Saravolatz}

St. John Hospital \& Medical Center

\section{Ashish Bhargava}

St. John Hospital \& Medical Center

\section{Research Article}

Keywords: Bloodstream infections, blood cultures, COVID-19, intensive care unit

Posted Date: August 18th, 2021

DOI: https://doi.org/10.21203/rs.3.rs-798567/v1

License: (c) (i) This work is licensed under a Creative Commons Attribution 4.0 International License. Read Full License 


\section{Abstract}

Objective: To describe the incidence, risk factors, and outcomes of bloodstream infections (BSIs) in patients with coronavirus disease 19 (COVID-19).

Methods: This was a single-center retrospective cohort study of adults admitted for COVID-19 with BSIs. Data were collected by electronic medical record review. BSIs were defined as positive blood cultures (BCs) with a known pathogen in one or more BCs or the same commensal organism in two or more BCs.

Results: Of 565 eligible patients, 290 (51.3\%) had BCs done, with 39 (13.4\%) having a positive result. In univariable analysis, male sex, black/African American race, admission from a facility, hemiplegia, altered mental status, and a higher Charlson Comorbidity Index were positively associated with a positive BC, whereas obesity and low systolic blood pressure (SBP) were negatively associated. Patients with positive BCs were more likely to have severe disease, be admitted to the Intensive Care Unit (ICU), require mechanical ventilation, have septic shock, and higher mortality. In multivariable logistic regression, factors that were independent predictors of a positive $B C$ were male sex $(O R=2.75, p=0.03)$, hypoalbuminemia ( $O R=3.3, p=0.01)$, ICU admission ( $O R=5.3, p<0.0001), S B P<100(O R=3.7, p=0.03)$ and having a procedure $(O R=10.5, p<0.0001)$. Patients with an abnormal chest $x$-ray on admission were less likely to have a positive $B C(O R=0.25, p=0.007)$.

Conclusions: We found that independent predictors of BSIs in COVID-19 patients included male sex, abnormal chest x-ray, hypoalbuminemia, admission to ICU, low SBP, and having a procedure during hospital stay.

\section{Background}

Coronavirus disease-19 (COVID-19) is caused by severe acute respiratory syndrome coronavirus 2 (SARSCoV2). By May 16, it had affected more than 168 million people worldwide and had caused more than 3.4 million deaths.[1] The disease can have a wide range of clinical presentations, commonly affecting the lungs, heart, kidneys, neurological, and gastrointestinal systems.[2] Severe presentations occur in up to $40 \%$ of hospitalized cases, commonly due to respiratory failure requiring mechanical ventilation and admission to an intensive care unit (ICU).[3]

Bacterial coinfections are common in respiratory viral infections. A previous study in Spanish adults admitted to the ICU during the $2009 \mathrm{H} 1 \mathrm{~N} 1$ influenza pandemic found that bacterial coinfection led to increased length of stay and resource utilization.[4] Similarly, bloodstream infections (BSIs) can increase morbidity and mortality when associated with viral infections. In hospitalized adults with COVID-19 from the US,[5, 6] UK,[7] Italy,[8] and Sweden,[9] the frequency of BSIs ranged from 2.5-7.9\%. In subjects with severe and critical illness, estimates of BSIs from the US[10], China,[11-14] and Italy[15] ranged from 0.9-49\%. Moreover, BSIs are considered a significant cause of morbidity and mortality in the US and Europe.[16] The current evidence highlights a wide range of frequencies of BSIs and the importance of identifying risk factors that can be targeted to decreased morbidity and mortality. Furthermore, minority 
patients such as black/African Americans are disproportionally affected by a higher incidence and greater disease severity of COVID-19.[2, 17]

We evaluated the incidence of BSIs in COVID-19 patients and described the potential risk factors associated with these infections in predominantly black/African American patients. We sought to identify risk factors that can help predict which patients will develop BSIs, to decrease morbidity and mortality.

\section{Methods}

Study subjects. We performed a retrospective, single-center historical cohort study of patients admitted for COVID-19 from March 8 to June 14 who had BSIs. BSIs were defined as a positive blood culture (BC) with a known pathogen in one or more BCs or the same commensal organism in two or more BCs. Inclusion criteria were adult patients (18 years and older) admitted for COVID-19 diagnosed with a positive reverse-transcriptase-polymerase-chain-reaction (RT-PCR) assay of a nasopharyngeal swab and had a BC done during their hospitalization. Exclusion criteria were adults who did not have BCs drawn during hospitalization or if the positive $\mathrm{BC}$ was due to an organism thought to be a contaminant. Clinical significance and source of BSIs were collected from infectious diseases (ID) consultation or progress notes. The study protocol was approved by the Institutional Review Board (IRB) of Ascension St. John Hospital.

Data collection. We collected data from the electronic medical record (EMR) via retrospective chart review. Demographic data collected included age, sex, race, nine-digit zip code, type of insurance, and admission source. Anthropometric data included weight, height, and body mass index (BMI). Obesity and morbid obesity were defined using the Centers for Disease Control and Prevention (CDC) guidelines.[18] We collected relevant medical comorbidities and calculated the Charlson Comorbidity Index, a validated weighted score established as a predictor of mortality.[19] COVID-19 severity was based on the World Health Organization (WHO) disease severity classification.[20] We collected data on vital signs and pulse oximetry on admission along with chest x-ray and chest computed tomography (CT) findings based on the official radiology report. Laboratory data included total white blood cell, lymphocyte, and platelet counts, C-reactive protein (CRP), serum creatinine, alanine aminotransferase (ALT), and aspartate aminotransferase (AST). We classified elevated and low laboratory values based on the hospital's cut points and categorized them as presented in Table 1. Elevated creatinine on admission was defined as an increase in serum creatinine $\geq 1.5$ times the baseline within seven days prior or an increase $\geq 0.3 \mathrm{mg} / \mathrm{dL}$ within 48 hours of admission.[21] We calculated the quick Sequential Organ Failure Assessment (qSOFA) on admission and used it to predict in-hospital sepsis-related mortality.[22] Septic shock was defined as hypotension requiring vasopressors. We defined central line as placement of any catheter (tunneled or non-tunneled) in any central vein during hospitalization. For procedures, we included any major emergent surgeries and any emergent endoscopic or percutaneous procedures done during the hospital stay. 
Table 1

Baseline characteristics of the study population by blood culture results

\begin{tabular}{|c|c|c|c|c|}
\hline Characteristic & $\begin{array}{l}\text { Total }(\mathrm{N}= \\
290)\end{array}$ & $\begin{array}{l}\text { Negative }(\mathrm{N}= \\
251)\end{array}$ & $\begin{array}{l}\text { Positive ( } \mathrm{N}= \\
\text { 39) }\end{array}$ & P-value \\
\hline Age, mean (SD) & $64.4 \pm 16.3$ & $64.4 \pm 16.5$ & $64.1 \pm 15.4$ & 0.90 \\
\hline Male, No. (\%) & $163(56.2)$ & $135(53.8)$ & $28(71.8)$ & 0.04 \\
\hline Race, No. (\%) & & & & 0.66 \\
\hline White & $61(21.1)$ & $53(21.2)$ & $8(20.5)$ & \\
\hline African American & $223(77.2)$ & $192(76.8)$ & $31(79.5)$ & \\
\hline Other & $5(1.7)$ & $5(2.0)$ & $0(0.0)$ & \\
\hline $\mathrm{BMI}$ in $\mathrm{kg} / \mathrm{m}^{2}$, mean (SD) & $31.8 \pm 9.2$ & $32.1 \pm 8.7$ & $29.8 \pm 11.7$ & 0.14 \\
\hline Obesity, No. (\%) & $149(52.3)$ & $134(54.5)$ & $15(38.5)$ & 0.06 \\
\hline Admission from facility, №. (\%) & $87(30.0)$ & $67(26.7)$ & $20(51.3)$ & 0.002 \\
\hline \multicolumn{5}{|l|}{ Comorbidities, No. (\%) } \\
\hline Hypertension & $214(73.8)$ & $187(74.5)$ & $27(69.2)$ & 0.49 \\
\hline Diabetes mellitus & $108(37.2)$ & $93(37.1)$ & $15(38.5)$ & 0.87 \\
\hline Congestive heart failure & $40(13.8)$ & $34(13.5)$ & $6(15.4)$ & 0.76 \\
\hline Prior myocardial infarction & $22(7.6)$ & $19(7.6)$ & $3(7.7)$ & 0.99 \\
\hline Cerebrovascular disease & $40(13.8)$ & $36(14.3)$ & $4(10.3)$ & 0.62 \\
\hline Dementia & $44(15.2)$ & $34(13.5)$ & $10(25.6)$ & 0.05 \\
\hline Hemiplegia & $22(7.6)$ & $15(6.0)$ & $7(17.9)$ & 0.009 \\
\hline Renal disease & $56(19.3)$ & $49(19.5)$ & $7(17.9)$ & 0.82 \\
\hline Liver disease & $11(3.8)$ & $8(3.2)$ & $3(7.7)$ & 0.17 \\
\hline Malignancy & $16(5.5)$ & $13(5.2)$ & $3(7.7)$ & 0.46 \\
\hline Chronic pulmonary disease ${ }^{a}$ & $74(25.5)$ & $65(25.9)$ & $9(23.1)$ & 0.71 \\
\hline $\begin{array}{l}\text { Charlson Comorbidity Index, median } \\
\text { (IQR) }\end{array}$ & $\begin{array}{l}1.0(0.0- \\
3.0)\end{array}$ & $1.0(0.0-2.0)$ & $2.0(1.0-3.0)$ & 0.01 \\
\hline
\end{tabular}

BMI: Body mass index; IQR: Interquartile range; qSOFA: quick Sequential Organ Failure Assessment; AST: Aspartate aminotransferase; ALT: Alanine aminotransferase.

Footnotes to Table 1

${ }^{\mathrm{a} C h r o n i c ~ p u l m o n a r y ~ d i s e a s e ~ i n c l u d e s ~ a s t h m a, ~ C O P D, ~ o r ~ b o t h . ~}$ 


\begin{tabular}{|c|c|c|c|c|}
\hline Characteristic & $\begin{array}{l}\text { Total }(\mathrm{N}= \\
290)\end{array}$ & $\begin{array}{l}\text { Negative }(\mathrm{N}= \\
251)\end{array}$ & $\begin{array}{l}\text { Positive (N = } \\
39)\end{array}$ & P-value \\
\hline Fever & $170(59.2)$ & $150(60.5)$ & $20(51.3)$ & 0.28 \\
\hline Altered mental status & $86(29.7)$ & $66(26.3)$ & $20(51.3)$ & 0.001 \\
\hline Fatigue & $101(36.1)$ & 96 (39.3) & $5(13.9)$ & 0.003 \\
\hline Shortness of breath & $210(73.9)$ & $189(76.8)$ & $21(55.3)$ & 0.005 \\
\hline Cough & $173(61.8)$ & $162(66.1)$ & $11(31.4)$ & $<.0001$ \\
\hline \multicolumn{5}{|l|}{ Vitals on admission, mean (SD) } \\
\hline Systolic blood pressure in $\mathrm{mmHg}$ & $132.2 \pm 25.1$ & $133.9 \pm 24.0$ & $121.4 \pm 29.2$ & 0.004 \\
\hline Diastolic blood pressure in $\mathrm{mmHg}$ & $74.3 \pm 16.7$ & $74.9 \pm 16.3$ & $70.7 \pm 19.1$ & 0.15 \\
\hline Oxygen saturation in \% & $93.0 \pm 8.1$ & $92.9 \pm 8.3$ & $93.7 \pm 6.9$ & 0.59 \\
\hline qSOFA & $1.0 \pm 0.7$ & $0.9 \pm 0.7$ & $1.3 \pm 0.8$ & 0.003 \\
\hline \multicolumn{5}{|l|}{$\begin{array}{l}\text { Laboratory findings on admission, } \\
\text { No. (\%) }\end{array}$} \\
\hline Leucopenia $\left(<4.0 \times 10^{9} / \mathrm{L}\right)$ & $27(9.3)$ & $19(7.6)$ & $8(20.5)$ & 0.01 \\
\hline Lymphopenia $\left(<1.0 \times 10^{9} / \mathrm{L}\right)$ & $156(54.2)$ & $135(54.0)$ & $21(55.3)$ & 0.88 \\
\hline Thrombocytopenia (< $\left.150 \times 10^{9} / \mathrm{L}\right)$ & $71(24.6)$ & $57(22.8)$ & $14(35.9)$ & 0.08 \\
\hline Hypoalbuminemia (<3.5 g/dL) & $139(49.1)$ & $111(45.3)$ & $28(73.7)$ & 0.001 \\
\hline Elevated AST (> 40 IU/L) & $160(58.2)$ & $136(57.4)$ & $24(63.2)$ & 0.50 \\
\hline Elevated ALT (> 40 IU/L) & $99(35.0)$ & $85(34.7)$ & $14(36.8)$ & 0.80 \\
\hline Elevated creatinine & $118(43.1)$ & $101(42.6)$ & $17(45.9)$ & 0.70 \\
\hline \multicolumn{5}{|l|}{ Treatment and procedures, №. (\%) } \\
\hline Steroids & $161(55.5)$ & $134(53.4)$ & $27(69.2)$ & 0.06 \\
\hline Central lines & $58(20.0)$ & $41(16.3)$ & $17(43.6)$ & $\begin{array}{l}<.0001 \\
0.00\end{array}$ \\
\hline Procedures & $9(3.1)$ & $4(1.6)$ & $5(12.8)$ & 0.003 \\
\hline \multicolumn{5}{|c|}{$\begin{array}{l}\text { BMI: Body mass index; IQR: Interquartile range; qSOFA: quick Sequential Organ Failure Assessment; } \\
\text { AST: Aspartate aminotransferase; ALT: Alanine aminotransferase. } \\
\text { Footnotes to Table } 1 \\
\text { achronic pulmonary disease includes asthma, COPD, or both. }\end{array}$} \\
\hline
\end{tabular}


Data Analysis. Descriptive statistics were computed to characterize the study groups. Continuous variables were reported as means and standard deviations or medians with interquartile range, and categorical variables were summarized as frequency distributions. Univariable analysis was done using Student's t-test for continuous normal and Mann-Whitney $U$ tests for non-normal variables. Chi-squared tests were used for categorical variables. For multivariable analysis, we used logistic regression to identify independent predictors of BSIs during the hospital stay, and we used stepwise forward regression to build the final model. Data were analyzed using SPSS v. 27.0, and a p-value less than 0.05 was indicated statistical significance.

\section{Results}

We had 565 patients admitted with a COVID-19 positive RT-PCR test between March 8 to June 14. Of those, 290 (51.3\%) patients had BCs drawn during their hospital stay, which served as the study population. Thirty-nine (13.4\%) had a positive BC. The average age ( \pm SD) of the analyzed population was $64.4 \pm 16.3$ years, with $56.2 \%$ males and $77.2 \%$ black/African American. The prevalence of obesity and morbid obesity were $52.3 \%$ and $18.6 \%$, respectively, and hypertension was the most common comorbidity (73.8\%). Compared to those with negative BCs, those with positive BCs were more likely to be male, black/African American, be admitted from a facility, have hemiplegia, have a higher Charlson Comorbidity Index, and less likely to be obese (Table 1). Most of the patients (78\%) had public insurance (Medicare or Medicaid), with no significant difference between groups.

The most common symptoms were shortness of breath, cough, and fever. Patients with positive BCs were significantly more likely to present with altered mental status and lower systolic blood pressure (SBP) but less likely to present with fatigue, shortness of breath, and cough, compared to those with negative BCs. (Table 1). Notably, $94.0 \%$ of the population had elevated CRP without significant difference between BC groups. Compared to those with negative BCs, those with positive BCs were more likely to present with leucopenia and hypoalbuminemia (Table 1).

In the positive BC group, patients had a lower incidence of abnormal chest x-ray on admission compared to those with negative results $(66.7 \%$ vs. $81.3 \%, p=0.04)$. Contrastingly, CT chest on admission was more likely to be abnormal in the positive $B C$ group compared to the negative group $(16.2$ vs. $6.6 \%, p=0.04)$.

Twenty percent $(n=58)$ and $3.1 \%(n=9)$ of the analyzed population had at least one central line placed and one procedure done during hospitalization. Both were significantly higher in the positive BC group (Table 1). Additionally, more than half of the population was treated with steroids during hospitalization (55.5\%), and those with positive BCs were more likely to receive steroids compared to those with negative BCs, approaching significance $(69.2 \%$. vs. $53.4 \%, p=0.06$ ) (Table 1$)$.

The prevalence of severe disease was $21.7 \%$, and around a third of patients were admitted to ICU (30\%) (Table 2). Compared to those with negative BCs, those with positive BCs were more likely to have severe disease, be admitted to ICU, require mechanical ventilation, have septic shock, and die $(64.1 \%$ vs. $32.7 \%$, $p<0.0001)$. 
Table 2

Treatment and clinical outcomes by blood culture status

\begin{tabular}{|lllll|}
\hline Characteristic & Total (N= 290) & Negative (N= 251) & Positive (N=39) & P-value \\
\hline Severity of Disease, No. (\%) & & & & 0.007 \\
\hline Mild & $113(39.0)$ & $95(37.8)$ & $18(46.2)$ & \\
\hline Moderate & $114(39.3)$ & $107(42.6)$ & $7(17.9)$ & \\
\hline Severe & $63(21.7)$ & $49(19.5)$ & $14(35.9)$ & \\
\hline ICU admission, No. (\%) & $87(30.0)$ & $64(25.5)$ & $23(59.0)$ & $<0.0001$ \\
\hline MV, No. (\%) & $86(29.7)$ & $63(25.1)$ & $23(59.0)$ & $<0.0001$ \\
\hline Septic shock, No. (\%) & $48(16.6)$ & $35(13.9)$ & $13(33.3)$ & 0.002 \\
\hline ARDS, No. (\%) & $32(11.0)$ & $25(10.0)$ & $7(17.9)$ & 0.14 \\
\hline AKI, No. (\%) & $125(43.1)$ & $104(41.4)$ & $21(53.8)$ & 0.15 \\
\hline Death & $107(36.9)$ & $82(32.7)$ & $25(64.1)$ & $<0.0001$ \\
\hline $\begin{array}{l}\text { ICU: Intensive Care Unit; MV: Mechanical Ventilation; ARDS: Acute Respiratory Distress Syndrome; AKI: } \\
\text { Acute Kidney Injury. }\end{array}$ & & & \\
\hline
\end{tabular}

In multivariable analysis, the variables initially introduced in the stepwise forward logistic regression included sex, Charlson Comorbidity Index, obesity, altered mental status, abnormal chest x-ray on admission, leucopenia, thrombocytopenia, hypoalbuminemia, CRP level, steroid treatment, admission from a facility, central line placement, procedures, severity of disease, admission to ICU, and SBP $\leq 100$ $\mathrm{mmHg}$. The final model included sex, abnormal chest x-ray on admission, hypoalbuminemia, admission to ICU, SBP $\leq 100 \mathrm{mmHg}$, and procedures. Notably, compared to those with negative BC, those with positive BCs during hospital stay were ten times more likely to have undergone a procedure, five times more likely to be admitted to ICU, twice as likely to be male, and three times more likely to have hypoalbuminemia and low SBP, and three times less likely to have an abnormal chest x-ray on admission (Table 3). 
Table 3

Multivariable analysis of predictors for positive blood

cultures

\begin{tabular}{|lll|}
\hline Characteristic & OR $(95 \%$ Cl $)$ & p-value \\
\hline Male sex & $2.75(1.10,6.87)$ & 0.03 \\
\hline Abnormal chest x-ray & $0.25(0.09,0.69)$ & 0.007 \\
\hline Hypoalbuminemia & $3.25(1.28,8.25)$ & 0.01 \\
\hline ICU admission & $5.30(2.24,12.51)$ & $<0.0001$ \\
\hline SBP $\leq 100$ mmHg & $3.73(1.22,11.43)$ & 0.02 \\
\hline Procedures & $10.48(1.47,74.59)$ & 0.02 \\
\hline ICU: Intensive Care Unit; SBP: Systolic Blood Pressure \\
\hline
\end{tabular}

\section{Discussion}

This study found a $13.4 \%$ incidence of BSIs in an urban population from Southeast Michigan, which is slightly higher than previously reported rates in patients from New York,[5, 6] Sweden,[9] London,[7] and Italy,[8] but lower than those from New Jersey.[10] The difference in estimates is likely related to variations in case definition and severity of disease. About a third of our population was admitted to the ICU, and the patients with positive BCs were more likely to be admitted to the ICU, have more severe disease, and require vasopressor support. Notably, admission to ICU and systolic hypotension were independent predictors of BSIs. Our findings are consistent with a study conducted in adults with severe COVID-19 from several centers from New Jersey[10] that found that patients with positive BCs were more likely to be admitted to ICU, have septic shock, and present with altered mental status than those with negative BCs. Notably, in univariable analysis, patients with positive BCs were more likely to present with altered mental status when compared to those with negative BCs.

Male sex and an increased number of comorbidities were associated with increased BSIs, but only male sex remained an independent predictor. A previous meta-analysis by Wu et al.[23] described an almost two-fold increased odds of severe COVID-19 in males compared to females. One of the proposed mechanisms for males having increased disease severity comes from the hypothesis that androgens facilitate SARS-CoV2 entry via the transmembrane protease serine 2 (TMPRSS2)-pathway. SARS-CoV2 spike proteins need trimming by TMPSS2 to bind to angiotensin-converting enzyme 2 (ACE2) receptors for entry to host cells. Androgens activate its receptor (AR), and the AR acts as a promoter of TMPRSS2 transcription resulting in facilitated host cell entry of SARS-CoV2[24]. We believe males have increased BSIs related to the increased severity of COVID-19.

Several laboratory abnormalities have been described in patients with COVID-19[2]. In our study, patients with positive BCs were more likely to present with leucopenia and hypoalbuminemia when compared to 
those with negative BCs. On multivariable analysis, only hypoalbuminemia was an independent predictor of BSIs. This is consistent with a previous metanalysis by Aziz et al.[25] that found hypoalbuminemia was associated with increased odds of severe COVID-19 vs. non-severe disease. Additionally, hypoalbuminemia has been associated with increased severity of illness in the context of viral, fungal, and bacterial infections, including COVID-19[26]. This evidence supports the role of hypoalbuminemia as a marker of more severe disease and can be considered a valuable predictor of BSIs in patients with COVID-19.

We also described that abnormal chest $\mathrm{x}$-ray on admission was less likely in the positive BC group when compared to the negative $\mathrm{BC}$ group, and this association remained significant in multivariable analysis. Contrastingly, CT chest on admission was more likely to be abnormal in the positive BC group compared to the negative group, but it was not a significant predictor in multivariable analysis. Similar to our results, a previous retrospective study of Chinese adults with COVID-19 reported abnormal findings in $59 \%$ of patients with a chest $\mathrm{x}$-ray[27]. We hypothesize that the abnormal CT chest did not remain a significant predictor in the multivariable analysis because of a low number of patients who had a CT chest done on admission compared to those who had a chest x-ray done (6 vs. 29). Additionally, an observational study conducted among adults from the UK described a lower sensitivity of the chest $\mathrm{x}$-ray when compared to CT chest for COVID-19 diagnosis using RT-PCR as gold standard[28], and we hypothesize that changes in CT chest occur earlier compared to xray, which could explain the discrepancy in our results.

Finally, we found that having at least one central line placement or a surgical procedure done during admission was associated with a positive $\mathrm{BC}$, but in multivariable analysis, only having a procedure was associated with higher odds of positive BCs, despite the small number of patients $(n=9)$. Surgical procedures are associated with a higher risk of BSIs but, to the best of our knowledge, no other studies have described an increased risk of BSIs associated with procedures in patients with COVID-19.

Our study is not without limitations. First, because of the small sample size, we were not able to stratify our results based on $\mathrm{BC}$ collection timing, and therefore our results also reflect the occurrence of hospitalrelated BSIs. Thus, we cannot know if BSIs resulted from greater disease severity or if having a BSI led to a more severe presentation. Moreover, due to the observational nature of our data, causal inference cannot be made. Because our study population is predominantly black/African American, and race can affect the association between COVID-19 and disease severity, our results may not be generalizable to a broader patient group. We did not find any association of steroid therapy with bloodstream infections, likely because of the low sample size. Finally, we did not have information on immunomodulatory therapies such as tocilizumab, which has been associated with increased risk of BSIs in critically ill patients with COVID-19.[29]

Our study has several strengths. We were able to identify predictors of bloodstream infections in patients hospitalized for COVID-19. Our results are consistent with previous studies, as stated above. We described potential predictors that can be used for early detection and to identify patients at risk, specifically, patients with COVID-19 admitted to ICU. Our population was predominantly black/African 
American, previously reported as disproportionally affected by a higher number of COVID-19 cases, and presenting with more severe disease[17]. We were also able to adjust for important risk factors such as placement of central lines and emergent procedures and described the role of procedures as a risk factor, despite the small sample size.

This historical cohort study found that independent predictors of bloodstream infections included male sex, hypoalbuminemia, admission to ICU, SBP $\leq 100 \mathrm{mmHg}$, abnormal chest $x$-ray, and procedures. We recommend further investigations to confirm these potential predictors that can lead to early identification and treatment of BSIs and reduce morbidity and mortality in adults with COVID-19, specifically identifying patients requiring emergency procedures as a high-risk group.

\section{Declarations}

\section{Ethics approval and consent to participate}

The study protocol was approved by the Institutional Review Board (IRB) of Ascension St. John Hospital

\section{Consent for publication}

Not applicable

\section{Availability of data and materials}

The datasets generated and/or analyzed during the current study are not publicly available due to HIPAA regulations, but are available from the corresponding author on reasonable request.

\section{Competing interests}

The authors declare that they have no competing interests.

\section{Funding}

No sources of funding were used to conduct this research

\section{Authors' contributions}

CVS: Study design, final manuscript writing, and editing

EAF: Data collection and revision of final manuscript

WZ: Data collection and revision of final manuscript

MS: Study design and final manuscript editing and revision

DY: Data collection and revision of final manuscript 
SS: Data analysis, collection, and final manuscript editing and revision

ML: Study design and final manuscript editing and revision

LS: Study design and final manuscript editing and revision

AB: Study design and final manuscript editing and revision

\section{Acknowledgements}

Not applicable

\section{References}

1. Johns Hopkins Coronavirus Resource Center. COVID-19 Map - Johns Hopkins Coronavirus Resource Center. Johns Hopkins Coronavirus Resource Center. 2020. https://coronavirus.jhu.edu/map.html. Accessed 23 Dec 2020.

2. Wiersinga WJ, Rhodes A, Cheng AC, Peacock SJ, Prescott HC. Pathophysiology, Transmission, Diagnosis, and Treatment of Coronavirus Disease 2019 (COVID-19): A Review. JAMA - Journal of the American Medical Association. 2020;324:782-93. doi:10.1001/jama.2020.12839.

3. Suleyman G, Fadel RA, Malette KM, Hammond C, Abdulla H, Entz A, et al. Clinical Characteristics and Morbidity Associated With Coronavirus Disease 2019 in a Series of Patients in Metropolitan Detroit. JAMA network open. 2020;3:e2012270. doi:10.1001/jamanetworkopen.2020.12270.

4. Martín-Loeches I, Sanchez-Corral A, Diaz E, Granada RM, Zaragoza R, Villavicencio C, et al. Communityacquired respiratory coinfection in critically III patients with pandemic 2009 influenza A(H1N1) virus. Chest. 2011;139:555-62. doi:10.1378/chest.10-1396.

5. Sepulveda J, Westblade LF, Whittier S, Satlin MJ, Greendyke WG, Aaron JG, et al. Bacteremia and blood culture utilization during covid-19 surge in New York City. J Clin Microbiol. 2020;58.

doi:10.1128/JCM.00875-20.

6. Goyal P, Choi JJ, Pinheiro LC, Schenck EJ, Chen R, Jabri A, et al. Clinical Characteristics of Covid-19 in New York City. N Engl J Med. 2020;382:2372-4. doi:10.1056/nejmc2010419.

7. Hughes S, Troise O, Donaldson H, Mughal N, Moore LSP. Bacterial and fungal coinfection among hospitalized patients with COVID-19: a retrospective cohort study in a UK secondary-care setting. Clin Microbiol Infect. 2020;26:1395-9. doi:10.1016/j.cmi.2020.06.025.

8. Ripa M, Galli L, Poli A, Oltolini C, Spagnuolo V, Mastrangelo A, et al. Secondary infections in patients hospitalized with COVID-19: incidence and predictive factors. Clin Microbiol Infect. 2020.

doi:10.1016/j.cmi.2020.10.021. 
9. Yu D, Ininbergs K, Hedman K, Giske CG, Strålin K, Özenci V. Low prevalence of bloodstream infection and high blood culture contamination rates in patients with COVID-19. PLoS One. 2020;15:e0242533. doi:10.1371/journal.pone.0242533.

10. Bhatt PJ, Shiau S, Brunetti L, Xie Y, Solanki K, Khalid S, et al. Risk Factors and Outcomes of Hospitalized Patients With Severe Coronavirus Disease 2019 (COVID-19) and Secondary Bloodstream Infections: A Multicenter Case-Control Study. Clin Infect Dis. 2020. doi:10.1093/cid/ciaa1748.

11. Zhang H, Zhang Y, Wu J, Li Y, Zhou X, Li X, et al. Risks and features of secondary infections in severe and critical ill COVID-19 patients. Emerg Microbes Infect. 2020;9:1958-64. doi:10.1080/22221751.2020.1812437.

12. Yang $X, Y u Y, X u$ J, Shu H, Xia J, Liu H, et al. Clinical course and outcomes of critically ill patients with SARS-CoV-2 pneumonia in Wuhan, China: a single-centered, retrospective, observational study. Lancet Respir Med. 2020;8:475-81. doi:10.1016/S2213-2600(20)30079-5.

13. Li X, Xu S, Yu M, Wang K, Tao Y, Zhou Y, et al. Risk factors for severity and mortality in adult COVID-19 inpatients in Wuhan. J Allergy Clin Immunol. 2020;146:110-8. doi:10.1016/j.jaci.2020.04.006.

14. Yu Y, Xu D, Fu S, Zhang J, Yang X, Xu L, et al. Patients with COVID-19 in 19 ICUs in Wuhan, China: A cross-sectional study. Crit Care. 2020;24. doi:10.1186/s13054-020-02939-x.

15. Cataldo MA, Tetaj N, Selleri M, Marchioni L, Capone A, Caraffa E, et al. Incidence of bacterial and fungal bloodstream infections in COVID-19 patients in intensive care: An alarming "collateral effect." J Glob Antimicrob Resist. 2020;23:290-1. doi:10.1016/j.jgar.2020.10.004.

16. Goto M, Al-Hasan MN. Overall burden of bloodstream infection and nosocomial bloodstream infection in North America and Europe. Clinical Microbiology and Infection. 2013;19:501-9. doi:10.1111/1469-0691.12195.

17. Bhargava A, Sharma M, Riederer K, Fukushima EA, Szpunar SM, Saravolatz L. Risk Factors for Inhospital Mortality from Coronavirus Disease 2019 Infection Among Black Patients-An Urban Center Experience. Clin Infect Dis. 2020. doi:10.1093/cid/ciaa1468.

18. Pi-Sunyer FX, Becker DM, Bouchard C, Carleton RA, Colditz GA, Dietz WH, et al. Clinical guidelines on the identification, evaluation, and treatment of overweight and obesity in adults: Executive summary. American Journal of Clinical Nutrition. 1998;68:899-917. doi:10.1093/ajcn/68.4.899.

19. Charlson ME, Pompei P, Ales KL, MacKenzie CR. A new method of classifying prognostic comorbidity in longitudinal studies: Development and validation. J Chronic Dis. 1987;40:373-83. doi:10.1016/00219681(87)90171-8.

20. WHO. Clinical management of COVID-19. WHO, Geneva. 2020. https://www.who.int/publications/i/item/clinical-management-of-covid-19. Accessed 20 Dec 2020. 
21. Khwaja A. KDIGO clinical practice guidelines for acute kidney injury. Nephron - Clinical Practice. 2012;120:c179-84. doi:10.1159/000339789.

22. Seymour CW, Liu VX, Iwashyna TJ, Brunkhorst FM, Rea TD, Scherag A, et al. Assessment of clinical criteria for sepsis for the third international consensus definitions for sepsis and septic shock (sepsis-3). JAMA - J Am Med Assoc. 2016;315:762-74. doi:10.1001/jama.2016.0288.

23. Wu X, Liu L, Jiao J, Yang L, Zhu B, Li X. Characterisation of clinical, laboratory and imaging factors related to mild vs. severe covid-19 infection: a systematic review and meta-analysis. Annals of Medicine. 2020;52:334-44. doi:10.1080/07853890.2020.1802061.

24. Mohamed MS, Moulin TC, Schiöth HB. Sex differences in COVID-19: the role of androgens in disease severity and progression. Endocrine. 2021;71:3-8. doi:10.1007/s12020-020-02536-6.

25. Aziz M, Fatima R, Lee-Smith W, Assaly R. The association of low serum albumin level with severe COVID-19: A systematic review and meta-analysis. Critical Care. 2020;24. doi:10.1186/s13054-02002995-3.

26. Wiedermann CJ. Hypoalbuminemia as Surrogate and Culprit of Infections. Int J Mol Sci. 2021;22. doi:10.3390/ijms22094496.

27. Guan W, Ni Z, Hu Y, Liang W, Ou C, He J, et al. Clinical Characteristics of Coronavirus Disease 2019 in China. N Engl J Med. 2020;382:1708-20. doi:10.1056/nejmoa2002032.

28. Borakati A, Perera A, Johnson J, Sood T. Diagnostic accuracy of X-ray versus CT in COVID-19: A propensity-matched database study. BMJ Open. 2020;10:42946. doi:10.1136/bmjopen-2020-042946.

29. Giacobbe DR, Battaglini D, Ball L, Brunetti I, Bruzzone B, Codda G, et al. Bloodstream infections in critically ill patients with COVID-19. Eur J Clin Invest. 2020;50. doi:10.1111/eci.13319. 